\title{
Minimally Invasive Surgical Approach for Spinal Canal Tumors-Technique Description and Experience from a Reference Center
}

\author{
Miguel Berbeo, Roberto Diaz, Juan Carlos Perez, Manuel Giraldo-Grueso, Santiago Gutierrez, \\ María Camila Villegas, Juan Acevedo, Oscar Feo Lee, Oscar Zorro, Juan Gomez, \\ Carlos Lindado, Pablo Harker, Juan Sardi
}

Hospital Universitario San Ignacio, Pontificia Universidad Javeriana, Bogotá, Colombia

Email: mberbeo@gmail.com, juanpmed@gmail.com

How to cite this paper: Berbeo, M., Diaz, R., Perez, J.C., Giraldo-Grueso, M., Gutierrez, S., Villegas, M.C., Acevedo, J., Lee, O.F., Zorro, O., Gomez, J., Lindado, C., Harker, P. and Sardi, J. (2017) Minimally Invasive Surgical Approach for Spinal Canal TumorsTechnique Description and Experience from a Reference Center. Journal of Cancer Therapy, 8, 268-277.

https://doi.org/10.4236/jct.2017.83022

Received: February 7, 2017

Accepted: March 19, 2017

Published: March 22, 2017

Copyright (ㅇ 2017 by authors and Scientific Research Publishing Inc. This work is licensed under the Creative Commons Attribution International License (CC BY 4.0).

http://creativecommons.org/licenses/by/4.0/

\begin{abstract}
Background: Spinal canal tumors are difficult to diagnose and treat. The traditional surgical approaches for attempting a complete excision of these lesions frequently involve big incisions and tissue dissection with high risk of postop instability and cerebrospinal fluid leakage. Also, there is a risk of neurological worsening, sometimes irreversible. Methods: We present our experience in a patient series with spinal canal tumors and describe the surgical approach with minimally invasive techniques (MIS). All of them were performed by the Neurosurgery team of the Hospital Universitario San Ignacio during the period of 2011-2016. Results: We reviewed forty patients with spinal canal tumors surgically treated with MIS techniques. 15 patients (37.5\%) had Meningioma diagnosis (complete resection in $11(73.3 \%)$, subtotal in $3(20 \%)$ and biopsy in one patient), 10 patients (25\%) with Schwannomas reached complete resection in $70 \%$ and subtotal in 30\%. 5 patients had spinal cord metastasis, with complete resection in 4 patients $(80 \%)$ and subtotal in $1(20 \%)$. Other included ependymoma, astrocytoma, and miscellaneous. No patient has had cerebrospinal fluid leakage and no postoperative fusion has been required. Conclusions: The minimally invasive approach allowed complete tumors removal in a high number of patients and good postoperative results. These findings are similar and in some cases, better than the reported with traditional techniques. This MIS technique provides encouraging results. It requires a wide learning curve and a high degree of surgical experience.
\end{abstract}

\section{Keywords}

Spinal Tumors, Minimally Invasive Spine Surgery, Surgical Technique 


\section{Introduction}

Spinal canal tumors are always a surgical challenge. The localization, size, and probable histology, are definitive factors to choose the surgical approach. Other factors are the in-situ patient's anatomical details and the instability present or predicted after surgery. They account for about $2 \%$ - $4 \%$ of all the tumors arising from the central nervous system (CNS) and a third of these are located intramedullary [1]. $69 \%$ of all primary tumors are benign and the 10 -year survival rate for malignant lesions is 64\%, mainly depending on the results of the surgical approach [1].

The presentation frequency of spinal canal tumors according to their histological subtype classification and order of frequency [2] is as follows: meningiomas, nerve sheath tumor and ependymomas, with a $33 \%, 27 \%$ and $21 \%$, respectively [3]. Because of the topographical distribution of spinal canal tumors [3], we believe that there are three facts of great importance in choosing an appropriate surgical technique meaning that: provides a low morbidity rate, great impact on the rate of complete resection of the tumor regardless of their location (intradural or extradural), and at the same time, a low postoperative instability. In our hospital, we have a widespread experience in developing and applying minimally invasive techniques to the spine surgery.

This study provides a case series report of spinal canal tumors and their surgical approach with minimally invasive techniques by the Neurosurgery team of the Hospital Universitario San Ignacio, Bogotá. We considered it to be of interest for the scientific community because we believe it will clarify the minimally invasion concepts as the basics for choosing procedures for the best possible outcome in this specific pathology.

\section{Methods and Materials}

\subsection{Population}

During the period of 2011-2016, forty patients were treated for spinal canal tumors in our institution (Hospital Universitario San Ignacio). The same surgeons performed all the surgeries (MB, $\mathrm{RD}$ ), the diagnosis was histologically confirmed and the same minimally invasive technique principles were used in all the cases.

We retrospectively review the medical records. Standard follow-up consisted of clinical visits at 1, 3 and 6 months after the surgery. Each follow-up the neurological status of the patients was documented.

\subsection{Inclusion Criteria}

1. Patients with spinal canal tumors candidates for minimally invasive surgery.

2. Karn of sky score higher than 70 .

3. Tomita score from 2 to 5 .

4. Tokuhashi score higher than 9 .

5. The tumor size should be smaller than one vertebral segment.

\subsection{Exclusion Criteria}

1. Patients with previous surgery at the same level. 
2. Patients who underwent radio surgery treatment.

3. Patients with unclear pathological diagnosis.

4. Lesions are not capable of being resected by an excisional approach.

\subsection{Surgical Technique}

A thoroughly analysis of the patient's images (contrast MRI and CT) was performed in the neurosurgical weekly meeting, and was performed again in the operating room, to confirm side and size of the incision, and to prevent possible risks (Figure 1). From the cervical to the lumbosacral levels, the patient always was placed in a prone position with protection pads in the pressure zones. We didn't use urinary catheter (a short surgical time always was expected). Intraoperative Multimodally Neuroelectrophysiological monitoring was used when indicated. All the procedures were performed under general anesthesia with bispectral monitoring, an arterial line was placed when needed, X-rays were used to identify the correct level and prevent mistakes. Sterile cleaning of the surgical field was performed as usually, and sterile clothing was placed. Guided by the previous radiological mark, a $3 \mathrm{~cm}$ paramedian incision was made (Figures 1-3). The dissection continues until the opening of the deep fascia. From this point, finger point blunt dissection was performed splitting the muscle fibers until the bone structures were identified (laminae, facet joints). Tubular dilators were placed sequentially. Finally, a tubular retractor was fixed on the operation table (Maxcess II, Nu-Vasive ${ }^{\circledR}, \mathrm{San}$ Diego, Ca, USA) and opened as necessary, usually one or two "clicks" (Figure 4). This process preserves totally the tendinous posterior tension band and decrease the possibility of instability secondary to soft tissues damage. Then the surgeon performed a bone resection as necessary, usually a hemilaminectomy, to obtain a good visual access to the lesion that was going to be resected. Bone resection of the facet joint medial third can provide a significant expansion of the visual field without generating an additional risk of instability. This approach allowed the view of the posterolateral, and when necessary the anterolateral content of the spinal canal. The dura was always opened with a surgical blade No. 11 and an angled microdissector. We don't use dural traction sutures. The dura was opened in a T-shape with the vertical cut directed to the tumor. To extend the exposure, the dural edges were retracted with bipolar electrocoagulation (Figure 5). The tumor removal was then performed under microscope magnification and classic microsurgery techniques (Figure 6). We first performed an internaldebulking of the tumor and then with gentle retraction we exposed its upper and lower limits to proceed with the final excision. Hemostasis was obtained with warm saline and little pieces of Surgicel ${ }^{\circledR}$ (Ethicon, Johnson \& Johnson) or hemostatic sponge. The dural closure was attained using a synthetic patch and fibrin sealant, no dural sutures were used. No drainage system was used. A scarce thick suture knots were placed in the muscle and fascia. The subcutaneous fat and the skin were closed as usually (Figure 3). A water-resistant bandage was placed over the incision.

\section{Results}

\section{Patient population}




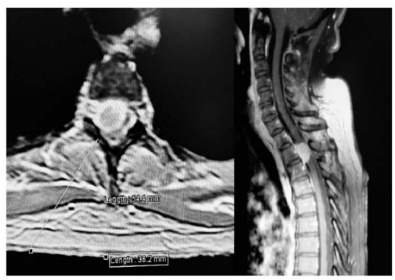

Figure 1. Surgical planning. An MRI T2 Sequence. Image shows a hyperintense mass with an intradural and dural location. The white line establishes the approach.

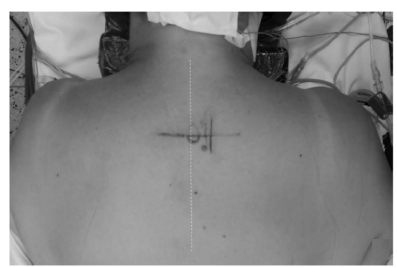

Figure 2. Surgical planning. A $3 \mathrm{~cm}$ paramedian incision.

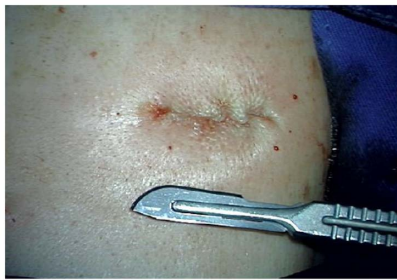

Figure 3. The final result of the minimally invasive technique, paramedian $3 \mathrm{~cm}$ incision.

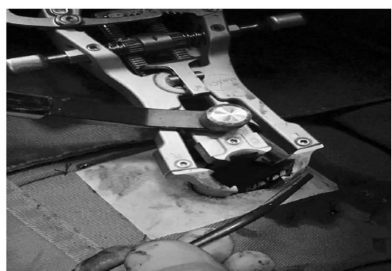

Figure 4. The tubular retractor fixed to the operation table.

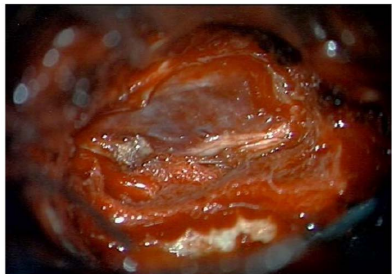

Figure 5. A microscopic view of an intradural, extramedullary mass. Meningioma.

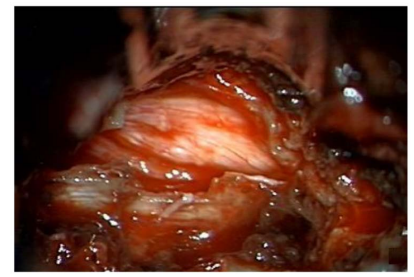

Figure 6. Microscopic view. The medulla after resection of an intradural, extramedullary mass. 
Forty patients with spinal cord tumors were reviewed. Clinical and operative variables are given in Table 1 . The main age at the time of surgery was 46 years (SD 15.2 yrs) 26 patients were female and 14 were male. Only two patients were pediatric (younger than $18 \mathrm{yrs}$ ) (Table 1). Clinical and operative variables are given in Table 2, 17 (42.5\%) patients presented with motor symptoms, 14 (35\%) with sensory symptoms and 3 (7.5\%) with bladder dysfunction.

The most common tumor location in our series was the thoracic spine with 20 (50\%) patients, followed by the cervical spine $12(30 \%)$ patients, then the lumbar spine $6(15 \%)$ and finally the craneo-cervical junction with 2 patients (5\%) (Table $1)$.

In our series, 15 (37.5\%) patients presented meningiomas. The resection was complete in $11(73.3 \%)$ patients, subtotal in $3(20 \%)$ and biopsy was done in one patient. 10 (25\%) patients were diagnosed with Schwannomas. A complete resection was achieved in $7(70 \%)$ patients, and subtotal in $3(30 \%)$ patients. 5 patients had spinal cord metastasis, a complete resection was carried out in $4(80 \%)$ patients, and subtotal resection in $1(20 \%)$ patient. The histology and resection results of the other tumors can be observed in Table 3 .

Additionally, 8 patients required preventive intraoperative arthrodesis, due to concern for potential spinal instability. The arthrodesis was planned preoperative by the surgeons. After the procedure 28 (70\%) patients reported subjective improvement of symptoms, 1 (2.5\%) patient described worsening of symptoms, and $11(27.5 \%)$ remained the same (Table 4$)$. Only three patients developed procedure related complications, we had one cerebrospinal fluid fistula, a spinal reperfusion injury and an abscess formation.

\section{Discussion}

Spinal canaltumors are about 10 to 15 times less common than cerebral tumors.

Table 1. Sociodemographic values of the patients.

\begin{tabular}{cc}
\hline SociodemographicValues & No. Patients (StD) \\
\hline Menage (yrs) & $46(15.2)$ \\
Male & 14 \\
Female & 26 \\
Mean Follow Up (yrs) & $1.68(0.82)$ \\
Pediatric Patients & 2 \\
\hline
\end{tabular}

Table 2. Clinical and operative variables.

\begin{tabular}{cc}
\hline Clinical or Radiographic Variable & No. of patients \\
\hline Clinical Parameters & 14 \\
Sensory symptoms & 17 \\
Motor weakness & 3 \\
Bladderdysfunction & \\
Spinelevels & 12 \\
Cervical spine & 20 \\
Thoracicspine & 6 \\
Lumbar spine & 2 \\
Craneo Cervical junction & \\
\hline
\end{tabular}


Table 3. Resection results. Partial, subtotal and complete resection of the spinal canal tumors. Percentage results showed in parenthesis.

\begin{tabular}{cccccc}
\hline Tumor & No. & $\%$ & Complete & Subtotal & Biopsy \\
\hline Meningioma & 15 & 37.5 & $11(73.3)$ & $3(20)$ & $1(6.6)$ \\
Ependymoma & 1 & 2.5 & 0 & $1(100)$ & 0 \\
Astrocytoma & 2 & 5 & $1(50)$ & $1(50)$ & 0 \\
Hemangioma & 1 & 2.5 & $1(100)$ & 0 & 0 \\
Metastasis & 5 & 12.5 & $4(80)$ & $1(20)$ & 0 \\
Schwannoma & 10 & 25 & $7(70)$ & $3(30)$ & 0 \\
Sarcoma & 2 & 5 & $2(100)$ & 0 & 0 \\
Nerveshealt tumor & 2 & 5 & 0 & $2(100)$ & 0 \\
Osteoma & 1 & 2.5 & $1(100)$ & 0 & 0 \\
Paraganglioma & 1 & 2.5 & 0 & $1(100)$ & 0 \\
\hline
\end{tabular}

Table 4. Examination findings after surgery. Number of patients and percentage.

\begin{tabular}{cc}
\hline Examination findings after OP & No. Patients \\
\hline Same & $11(27.5)$ \\
Worse & $1(2.5)$ \\
Better & $28(70)$
\end{tabular}

They represent $2 \%-4 \%$ of all CNS tumors and are classified according to their anatomical location into 3 subtypes: intramedullary (originated from medullary parenchyma), extraaxial intradural (located in the meningeal covering but aren't part of the medullary parenchyma) and extradural (outside of the meningeal covering but compromise the medullary canal) [4].

The symptoms of the spinal canaltumors vary depending on the location of the tumor. In a recent case series report it was documented that the main complaint was pain in a $72 \%$ of patients, followed by motor deficit in a $55 \%$ and sensitive symptoms in $39 \%$ of the cases [5]. In our series, $42.5 \%$ of the patients presented with a motor deficit and $35 \%$ with sensitive symptoms.

Within the primary tumors, the most common subtypes are ependymomas in a $45 \%$ followed by astrocytomas with $40 \%$ [4]. Although there is a high survival rate, the tumor free period largely depends on the surgical approach. Astrocytomas are considered to have the worse prognosis amongst these types of lesions since their resection margin doesn't allow a complete resection in most cases, conditioning a recurrence rate of $47 \%$ [6] [7] [8]. In our case series, of two patients with astrocytomas, we accomplished one complete resection and one subtotal resection. For ependymomas, there was only one case for which a subtotal resection was performed. The Mayo Clinic published a case series of medullary astrocytomas, they accomplished a complete resection in $16 \%$ of the cases and a subtotal resection in $25 \%$ of the patients [9].

In extramedullary, intradural tumors, the surgical approach determines the 
prognosis independent of its origin, whether it is a benign tumor (meningiomas, schwannoma or neurofibroma) or a more aggressive one. Studies report that the outcomes for minimally invasive and open surgery are comparable [10]. In our series of minimally invasive surgery, meningioma was the most frequent tumor (37.5\%) with a complete resection rate of $73.3 \%$ and a subtotal resection of $20 \%$. We had 10 cases of schwannomas (25\%) with a success rate for complete resection of $70 \%$ and $30 \%$ for subtotal resection. Other case series have shown similar results. The Helsinki University accomplished a complete resection in $86 \%$ of the Shwannomas and a subtotal resection in $11 \%$ patients [11] and the University of Seoul achieved a complete resection in $84.2 \%$ of the patients with Meningiomas and a partial resection in $15.8 \%$. These results were accomplished with traditional surgery approaches [12] [13].

Metastatic lesions represented $12.5 \%$ of all tumors and we accomplished a complete resection rate of $80 \%$ and subtotal resection of $20 \%$. In the treatment of nonprimary tumors such as metastasis, conventional techniques with decompression and involvement of posterior vertebral elements described in case series reports have shown clinical improvement and stabilization in at least $50 \%$ of the cases. It is of great importance to highlight the fact that prognosis of any spinal cord pathology, whether it is metastatic or primary origin, it's determined by the possibility of a bloc resection [14].

A case series of 142 patients with post operative follow-up reported that $78 \%$ of the patients had symptomatic improvement, $15 \%$ remain the same and $7 \%$ referred worsening. In our series 28 (70\%) patients reported subjective improvement, 1 (2.5\%) patient described worsening of symptoms, and 11 (27.5\%) remained the same [15].

The mainstream treatment of spinal canaltumors is surgical resection. Several surgical approaches have been proposed aiming at a lower morbimortality and a greater postoperative stability of the column. Minimally invasive techniques have been described, considering a hemilaminectomy with preservation of supraspinatus and infraspinatus ligaments and contralateral paravertebral muscles. Cost effective studies have compared this surgical approach vs. traditional surgical procedures and have shown a reduction in hospital costs of about $30 \%$, given the reduction in hospitalization days, reintervention procedures and of complications [16].

Spinal canaltumors are uncommon if considered within the whole range of CNS pathologies, yet they represent an important source of disability and morbidity that may affect the patients' life quality. Taking this into account, it is also important to know the possible risks that this approach may bring with, as well as its advantages compared with open procedures. A non-described advantage of the paramedian minimally invasive approach to intra canal tumors is the angled view of the canal and its contents. After limited bone resection of the lamina and facet joint, the ventrolateral aspect of the dura is exposed allowing to the surgeon to navigate into the anteriorly placed canal tumors directly. This path could be safer because the spinal cord manipulation is lower and the risk of neural damage decreases. 
In a retrospective study from the University of Stanford where minimally invasive technique was compared with open surgery, there were no significant differences in the presentation of complications $(p=0.50)$, however there was a significant decrease in the amount of estimated blood loss in minimally invasive surgery $(p<0.01)$. Also, it was demonstrated that there is no difference in the rate of total resection of the tumor, suggesting good results for both techniques. Minimally invasive surgery did demonstrate a decrease in the mean hospital stay when compared with open surgery. In relation to postoperative complications, the spinal fluid leak was the most frequent with a significant decrease in minimally invasive surgery with a rate of $3.7 \%$ compared to $16.7 \%$ in the open group in this study $(p=0.03)$ [17].

In our hospital, minimally invasive surgery only needs one day of clinical surveillance after the procedure (\$300 US approximate) added to the global intra and perioperative costs. Special intraoperative supplies like dural grafts and fibrin sealants don't make differences in cost because they are used in open and minimally invasive approaches in a similar way. We don't use ICU stay for this surgery. Traditional techniques usually need 3 - 4 days of clinical surveillance ( $\$ 900$ - $\$ 1200$ US approximate). A cost effectiveness analysis from the Università Politecnicadelle Marche, Umberto I General Hospital, Ancona, Italia, concludes that the minimally invasive compared to open technique, reduces muscular damage, blood loss, postoperative pain and hospital stay while achieving comparable results in both techniques. The reduction in postoperative complications seen in minimally invasive surgery is most likely what makes these approaches more cost effective compared with open technique [18] [19].

\section{Conclusion}

Spinal canal tumors are uncommon if considered within the whole range of CNS pathologies; yet, they represent an important source of disability and morbidity that may affect the patients' life quality. The minimally invasive approach allows a complete tumor removal and better postoperative results. This technique should be encouraged. However, it requires a high degree of surgical experience.

\section{Disclaimer}

The authors report no conflict of interest concerning this paper.

\section{References}

[1] Schellinger, K.A., Propp, J.M., Villano, J.L. and McCarthy, B.J. (2008) Descriptive Epidemiology of Primary Spinal Cord Tumors. Journal of Neuro-Oncology, 87, 173-179. https://doi.org/10.1007/s11060-007-9507-z

[2] National Cancer Institute SSB (2007) Cancer Incidence-Surveillance, Epidemiology, and End Results (SEER) Registries Research Data [Internet]. Surveillance, Epidemiology, and End Results (SEER) Program (http://www.seer.cancer.gov). http://seer.cancer.gov/data/metadata.html

[3] Duong, L.M., McCarthy, B.J., McLendon, R.E., Dolecek, T.A., Kruchko, C., Douglas, L.L., et al. (2012) Descriptive Epidemiology of Malignant and Nonmalignant Prima- 
ry Spinal Cord, Spinal Meninges, and Cauda Equinatumors, United States, 20042007. Cancer, 118, 4220-4227. https://doi.org/10.1002/cncr.27390

[4] Harrop, J.S., Ganju, A., Groff, M. and Bilsky, M. (2009) Primary Intramedullary Tumors of the Spinal Cord. Spine, 34, S69-S77.

https://doi.org/10.1097/BRS.0b013e3181b95c6f

[5] Karikari, I.O., Nimjee, S.M., Hodges, T.R., Cutrell, E., Hughes, B.D., Powers, C.J., et al. (2015) Impact of Tumor Histology on Resectability and Neurological Outcome in Primary Intramedullary Spinal Cord Tumors: A Single-Center Experience with 102 Patients. Neurosurgery, 76, S4-S13. https://doi.org/10.1227/01.neu.0000462073.71915.12

[6] Tobin, M.K., Geraghty, J.R., Engelhard, H.H., Linninger, A.A. and Mehta, A.I. (2015) Intramedullary Spinal Cord Tumors: A Review of Current and Future Treatment Strategies. Neurosurgical Focus, 39, E14.

http://www.ncbi.nlm.nih.gov/pubmed/26235012 https://doi.org/10.3171/2015.5.FOCUS15158

[7] Waldron, J.N., et al. (1993) Spinal Cord Ependymomas: A Retrospective Analysis of 59 Cases. Journal of Radiation Oncology, 27, 223-229.

https://doi.org/10.1016/0360-3016(93)90231-J

[8] Fontes, R.B.V., Wewel, J.T. and O’Toole, J.E. (2016) Perioperative Cost Analysis of Minimally Invasive vs. Open Resection of Intradural Extramedullary Spinal Cord Tumors. Neurosurgery, 78, 531-539. https://doi.org/10.1227/NEU.0000000000001079

[9] Takami, T., Naito, K., Yamagata, T. and Ohata, K. (2015) Surgical Management of Spinal Intramedullary Tumors: Radical and Safe Strategy for Benign Tumors. Neurologia Medico-Chirurgica (Tokyo), 55, 317-327.

[10] Minehan, K.J., Brown, P.D., Scheithauer, B.W., Krauss, W.E. and Wright, M.P. (2009) Prognosis and Treatment of Spinal Cord Astrocytoma. International Journal of Radiation Oncology ${ }^{\star}$ Biology ${ }^{\star}$ Physics, 73, 727-733. https://doi.org/10.1016/j.ijrobp.2008.04.060

[11] Seppälä, M.T., Haltia, M.J., Sankila, R.J., Jääskeläinen, J.E. and Heiskanen, O. (1995) Long-Term Outcome after Removal of Spinal Schwannoma: A Clinicopathological Study of 187 Cases. Journal of Neurosurgery, 83, 621-626.

http://www.ncbi.nlm.nih.gov/pubmed/7674010 https://doi.org/10.3171/jns.1995.83.4.0621

[12] Kaloostian, P.E., Zadnik, P.L., Etame, A.B., Vrionis, F.D., Gokaslan, Z.L. and Sciubba, D.M. (2014) Surgical Management of Primary and Metastatic Spinal Tumors. Cancer Control, 21, 133-139.

[13] Yoon, S.H., Chung, C.K. and Jahng, T.A. (2007) Surgical Outcome of Spinal Canal Meningiomas. Journal of Korean Neurosurgical Society, 42, 300-304. https://doi.org/10.3340/jkns.2007.42.4.300

[14] Harel, R. and Angelov, L. (2010) Spine Metastases: Current Treatments and Future Directions. European Journal of Cancer, 46, 2696-2707. https://doi.org/10.1016/j.ejca.2010.04.025

[15] Wong, A.P., Lall, R.R., Dahdaleh, N.S., Lawton, C.D., Smith, Z.A., Wong, R.H., et al. (2015) Comparison of Open and Minimally Invasive Surgery for Intradural-Extramedullary Spine Tumors. Neurosurgical Focus, 39, E11. http://thejns.org/doi/abs/10.3171/2015.5.FOCUS15129

[16] Vertuani, S., Nilsson, J., Borgman, B., Buseghin, G., Leonard, C., Assietti, R. and Quraishi, N.A. (2015) A Cost-Effectiveness Analysis of Minimally Invasive versus Open Surgery Techniques for Lumbar Spinal Fusion in Italy and the United King- 
dom. Value in Health, 18, 810-816. https://doi.org/10.1016/j.jval.2015.05.002

[17] Iacoangeli, M., Gladi, M., Di Rienzo, A., Dobran, M., Alvaro, L., Nocchi, N., et al. (2012) Minimally Invasive Surgery for Benign Intradural Extramedullary Spinal Meningiomas: Experience of a Single Institution in a Cohort of Elderly Patients and Review of the Literature. Clinical Interventions in Aging, 7, 557-564. https://doi.org/10.2147/CIA.S38923

[18] Nzokou, A., Weil, A.G. and Shedid, D. (2013) Minimally Invasive Removal of Thoracic and Lumbar Spinal Tumors Using a Nonexpandable Tubular Retractor: Clinical Article. Journal of Neurosurgery: Spine, 19, 708-715.

http://thejns.org/doi/pdf/10.3171/2013.9.SPINE121061 https://doi.org/10.3171/2013.9.SPINE121061

[19] Tredway, T.L. (2014) Minimally Invasive Approaches for the Treatment of Intramedullary Spinal Tumors. Neurosurgery Clinics of North America, 25, 327-336. https://doi.org/10.1016/j.nec.2013.12.010

\section{Submit or recommend next manuscript to SCIRP and we will provide best} service for you:

Accepting pre-submission inquiries through Email, Facebook, LinkedIn, Twitter, etc. A wide selection of journals (inclusive of 9 subjects, more than 200 journals)

Providing 24-hour high-quality service

User-friendly online submission system

Fair and swift peer-review system

Efficient typesetting and proofreading procedure

Display of the result of downloads and visits, as well as the number of cited articles Maximum dissemination of your research work

Submit your manuscript at: http://papersubmission.scirp.org/

Or contact jct@scirp.org 